\title{
NEW SEVERAL INTEGRAL INEQUALITIES
}

\author{
WAAD SULAIMAN
}

Abstract. Several new integral inequalities are presented.

\section{Introduction}

In [3] the following result was proved

Theorem 1.1. If $f \geq 0$ is continuous function on $[a, b]$ such that

$$
\int_{x}^{1} f(t) d t \geq \int_{x}^{1} t d t, \quad \forall x \in[0,1]
$$

then

$$
\int_{0}^{1} f^{\alpha+1}(x) d x \geq \int_{0}^{1} x^{\alpha} f(x) d x, \quad \forall \alpha>0,
$$

and the following question was posed

If $f$ satisfies the above assumption, under what additional assumption can one claim that

$$
\int_{0}^{1} f^{\alpha+\beta}(x) d x \geq \int_{0}^{1} x^{\alpha} f^{\beta}(x) d x, \quad \forall \alpha, \beta>0 .
$$

The following result as well, was achieved in [2].

Theorem 1.2. If $f \geq 0$ is a continuous function on $[0, b]$ satisfying

$$
\int_{x}^{b} f^{\alpha}(t) d t \geq \int_{x}^{b} t^{\alpha} d t, \quad b>0, \quad \forall x \in[0, b]
$$

then

$$
\int_{0}^{b} f^{\alpha+\beta}(x) d x \geq \int_{0}^{b} x^{\alpha} f^{\beta}(x) d x, \quad \forall \beta>0 .
$$

In his role, Hoang [1] generalized the previous results by introducing the following

2010 Mathematics Subject Classification. $26 \mathrm{D} 15$.

Key words and phrases. Integral inequality. 
Theorem 1.3. Suppose $f, g \in L^{1}[a, b], f, g \geq 0, g$ is non-decreasing. If

$$
\int_{x}^{b} f(t) d t \geq \int_{x}^{b} g(t) d t, \quad \forall x \in[a, b],
$$

then

$$
\int_{a}^{b} f^{\alpha+\beta}(x) d x \geq \int_{a}^{b} f^{\alpha}(x) g^{\beta}(x) d x, \forall \alpha, \beta \geq 0, \alpha+\beta \geq 1 .
$$

\section{Results}

We prove the following

Theorem 2.1. Let $f, g \geq 0$, and defined on $[a, b]$.Define

$$
F^{\alpha}(x)=\int_{a}^{x} f^{\alpha}(t) d t, \quad G^{\alpha}(x)=\int_{a}^{x} g^{\alpha}(t) d t .
$$

If

$$
\int_{x}^{b} F^{\beta}(t) d t \geq \int_{x}^{b} G^{\beta}(t) d t, \forall x \in[a, b]
$$

then

for all $\alpha, \beta \geq 0$.

$$
\int_{a}^{b} F^{\alpha+\beta}(x) d x \geq \int_{a}^{b} F^{\alpha}(x) G^{\beta}(x) d x,
$$

Proof. We have

$$
\begin{aligned}
\int_{a}^{b} F^{\beta}(x) G^{\alpha}(x) d x & =\int_{a}^{b} F^{\beta}(x) \int_{a}^{x} g^{\alpha}(t) d t d x \\
& =\int_{a}^{b} g^{\alpha}(t) \int_{t}^{b} F^{\beta}(x) d x d t \\
& \geq \int_{a}^{b} g^{\alpha}(t) \int_{t}^{b} G^{\beta}(x) d x d t \\
& =\int_{a}^{b} G^{\beta}(x) \int_{a}^{x} g^{\alpha}(t) d t d x \\
& =\int_{a}^{b} G^{\alpha+\beta}(x) d x .
\end{aligned}
$$

Applying the Arithmetic-geometric inequality, we have for $\beta, \alpha \geq 0$

$$
F^{\alpha}(x) G^{\beta}(x) \leq \frac{\alpha}{\alpha+\beta} F^{\alpha+\beta}(x)+\frac{\beta}{\alpha+\beta} G^{\alpha+\beta}(x) .
$$

Integrating the above inequality and using 2.3 yields

$$
\int_{a}^{b} F^{\alpha}(x) G^{\beta}(x) d x \leq \frac{\alpha}{\alpha+\beta} \int_{a}^{b} F^{\alpha+\beta}(x) d x+\frac{\beta}{\alpha+\beta} \int_{a}^{b} G^{\alpha+\beta}(x) d x
$$




$$
\leq \frac{\alpha}{\alpha+\beta} \int_{a}^{b} F^{\alpha+\beta}(x) d x+\frac{\beta}{\alpha+\beta} \int_{a}^{b} F^{\alpha}(x) G^{\beta}(x) d x,
$$

and hence

$$
\int_{a}^{b} F^{\alpha+\beta}(x) d x \geq \int_{a}^{b} F^{\alpha}(x) G^{\beta}(x) d x .
$$

Theorem 2.2. Let $f, g \geq 0$, and defined on $[a, b]$. Define

$$
F^{\alpha}(x)=\int_{a}^{x} f^{\alpha}(t) d t, \quad G^{\alpha}(x)=\int_{a}^{x} g^{\alpha}(t) d t .
$$

If

$$
\int_{x}^{b} F(t) d t \geq \int_{x}^{b} G(t) d t, \quad \forall x \in[a, b]
$$

then

$$
\int_{a}^{b} F^{\alpha+\beta}(x) d x \geq \int_{a}^{b} F^{\alpha}(x) G^{\beta}(x) d x,
$$

for all $\alpha \geq 1, \beta \geq 0$.

Proof. Making use of 2.3 with $\beta=1$, gives

$$
\int_{a}^{b} F(x) G^{\alpha}(x) d x \geq \int_{a}^{b} G^{\alpha+1}(x) d x .
$$

By the AG inequality, we have for $\alpha \geq 1$,

$$
\frac{1}{\alpha} F^{\alpha}(x)+\frac{\alpha-1}{\alpha} G^{\alpha}(x) \geq F(x) G^{\alpha-1}(x) .
$$

Integrating the above inequality

$$
\frac{1}{\alpha} \int_{a}^{b} F^{\alpha}(x) d x+\frac{\alpha-1}{\alpha} \int_{a}^{b} G^{\alpha}(x) d x \geq \int_{a}^{b} F(x) G^{\alpha-1}(x) d x,
$$

which implies with the use of 2.6

$$
\begin{aligned}
\frac{1}{\alpha} \int_{a}^{b} F^{\alpha}(x) d x & \geq \int_{a}^{b} F(x) G^{\alpha-1}(x) d x-\frac{\alpha-1}{\alpha} \int_{a}^{b} G^{\alpha}(x) d x \\
& \geq \int_{a}^{b} G^{\alpha}(x) d x-\frac{\alpha-1}{\alpha} \int_{a}^{b} G^{\alpha}(x) d x \\
& =\frac{1}{\alpha} \int_{a}^{b} G^{\alpha}(x) d x, \quad \alpha \geq 1 .
\end{aligned}
$$

Again, by the AG inequality,

$$
F^{\alpha}(x) G^{\beta}(x) \leq \frac{\alpha}{\alpha+\beta} F^{\alpha+\beta}(x)+\frac{\beta}{\alpha+\beta} G^{\alpha+\beta}(x) .
$$

Since, by 2.7, we have for $\alpha \geq 1, \beta>0$,

$$
\int_{a}^{b} F^{\alpha+\beta}(x) d x \geq \int_{a}^{b} G^{\alpha+\beta}(x) d x,
$$

the result follows by integrating 2.8 and making use of 2.9. 
Corollary 2.3. Let $f, g \geq 0$, and defined on $[a, b]$. Define

$$
F^{\alpha}(x)=\int_{a}^{x} f^{\alpha}(t) d t, \quad G^{\alpha}(x)=\int_{a}^{x} g^{\alpha}(t) d t .
$$

If 2.4 is satisfied, then

$$
2 \int_{a}^{b} F^{\alpha}(x) G^{\beta}(x) d x \leq \int_{a}^{b}\left(F^{2 \alpha}(x)+F^{2 \beta}(x)\right) d x, \forall \beta \geq 1 / 2 .
$$

In particular, if $F^{\beta}(x) \leq F^{\alpha}(x)$, then

$$
\int_{a}^{b} F^{\alpha}(x) G^{\beta}(x) d x \leq \int_{a}^{b} F^{2 \alpha}(x) d x .
$$

Proof. As

$$
\left(F^{\alpha}(x)-G^{\beta}(x)\right)^{2} \geq 0,
$$

opening and integrating gives

$$
2 \int_{a}^{b} F^{\alpha}(x) G^{\beta}(x) d x \leq \int_{a}^{b}\left(F^{2 \alpha}(x)+G^{2 \beta}(x)\right) d x .
$$

By 2.7,

$$
\int_{a}^{b} F^{2 \beta}(x) d x \geq \int_{a}^{b} G^{2 \beta}(x) d x
$$

then, the corollary follows.

The other way round direction follows from the coming result

Theorem 2.4. Let $f, g \geq 0$, and defined on $[a, b]$.Define

$$
F^{\alpha}(x)=\int_{a}^{x} f^{\alpha}(t) d t, \quad G^{\alpha}(x)=\int_{a}^{x} g^{\alpha}(t) d t, \quad \alpha \in R .
$$

If

$$
\int_{a}^{b} F^{\beta}(x) d x \leq \int_{a}^{b} G^{\beta}(x) d x, \quad \forall x \in[a, b]
$$

then

$$
\int_{a}^{b} F^{\beta-\alpha}(x) d x \leq \int_{a}^{b} F^{\beta}(x) G^{-\alpha}(x) d x
$$

for all $\beta>\alpha>0$.

Proof. We have

$$
\int_{a}^{b} F^{\beta}(x) G^{-\alpha}(x) d x=\int_{a}^{b} F^{\beta}(x) \int_{a}^{x} g^{-\alpha}(t) d t d x
$$




$$
\begin{aligned}
& =\int_{a}^{b} g^{-\alpha}(t) \int_{t}^{b} F^{\beta}(x) d x d t \\
& \leq \int_{a}^{b} g^{-\alpha}(t) \int_{t}^{b} G^{\beta}(x) d x d t \\
& =\int_{a}^{b} G^{\beta}(x) \int_{a}^{x} g^{-\alpha}(t) d t d x \\
& =\int_{a}^{b} G^{\beta-\alpha}(x) d x .
\end{aligned}
$$

Applying the AG inequality, we have for $0<\alpha<\beta$,

$$
F^{\beta}(x) G^{-\alpha}(x) \geq \frac{\beta}{\beta-\alpha} F^{\beta-\alpha}(x)-\frac{\alpha}{\beta-\alpha} G^{\beta-\alpha}(x) .
$$

Integrating the above inequality, and using 2.14 , we obtain

$$
\begin{aligned}
\int_{a}^{b} F^{\beta}(x) G^{-\alpha}(x) d x & \geq \frac{\beta}{\beta-\alpha} \int_{a}^{b} F^{\beta-\alpha}(x) d x-\frac{\alpha}{\beta-\alpha} \int_{a}^{b} G^{\beta-\alpha}(x) d x \\
& \geq \frac{\beta}{\beta-\alpha} \int_{a}^{b} F^{\beta-\alpha}(x) d x-\frac{\alpha}{\beta-\alpha} \int_{a}^{b} F^{\beta}(x) G^{-\alpha}(x) d x,
\end{aligned}
$$

and hence 2.13 follows.

Theorem 2.5. Let $f, g \geq 0$, and defined on $[a, b]$.Define

$$
F^{\alpha}(x)=\int_{x}^{b} f^{\alpha}(t) d t, \quad G^{\alpha}(x)=\int_{x}^{b} g^{\alpha}(t) d t .
$$

If

$$
\int_{a}^{x} F^{\beta}(t) d t \geq \int_{a}^{x} G^{\beta}(t) d t, \forall x \in[a, b]
$$

then

$$
\int_{a}^{b} F^{\alpha+\beta}(x) d x \geq \int_{a}^{b} F^{\alpha}(x) G^{\beta}(x) d x,
$$

for all $\alpha, \beta \geq 0$.

Proof. We have

$$
\begin{aligned}
\int_{a}^{b} F^{\beta}(x) G^{\alpha}(x) d x & =\int_{a}^{b} F^{\beta}(x) \int_{x}^{b} g^{\alpha}(t) d t d x \\
& =\int_{a}^{b} g^{\alpha}(t) \int_{a}^{t} F^{\beta}(x) d x d t \\
& \geq \int_{a}^{b} g^{\alpha}(t) \int_{a}^{t} G^{\beta}(x) d x d t \\
& =\int_{a}^{b} G^{\beta}(x) \int_{x}^{b} g^{\alpha}(t) d t d x
\end{aligned}
$$




$$
=\int_{a}^{b} G^{\alpha+\beta}(x) d x
$$

The rest can be achieved exactly as it has been done in theorem 2.1, and therefore it is omitted.

\section{References}

[1] N. S. Hoang, Notes on an inequalities, J. Ineq. Pure Appl. Math., 9(2009), Art. 42.

[2] W. J. Liu, C. C. Li and J. W. Dong, On an problem concerning an integral inequality, J. Ineq. Pure Appl. Math., 8 (2007), Art. 74.

[3] Q. A. Ngo, D. D. Thang, T. T. Dat and D. A. Tuan, Notes on an integral inequality, J. Pure Appl. Math., 7(4) (2006), Art. 120.

Department of Computer Engineering, College of engineering, University of Mosul, Iraq.

E-mail: waadsulaiman@hotmail.com 